\title{
Well-posedness for a semilinear heat-wave type equation in Besov-Morrey spaces
}

Marcelo F. de Almeida ${ }^{1}$

Departamento de Matemática, UFS, São Cristóvão, SE

Juliana C. Precioso ${ }^{2}$

Departamento de Matemática, UNESP, São José do Rio Preto, SP

\begin{abstract}
This work considers a semilinear integro-differential equation of Volterra type which interpolates semilinear heat and wave equations. Global existence of solutions is showed in spaces of Besov type based in Morrey spaces, namely BesovMorrey spaces. Our initial data is larger than the previous works and our results provide a maximal existence class for semilinear interpolated heat-wave equation.
\end{abstract}

Keywords. Fractional partial differential equation, Riemann-Liouville derivative, BesovMorrey spaces

\section{Introduction}

In this work we consider the semilinear integro-partial differential equation in $\mathbb{R}^{n}$, which reads as

$$
\left\{\begin{array}{l}
u_{t}=\int_{0}^{t} r_{\alpha}(t-s)[P(D) u(s)+f(u(s))] d s,\left(x \in \mathbb{R}^{n} \text { and } t>0\right) \\
u(0, x)=u_{0}(x), x \in \mathbb{R}^{n}
\end{array}\right.
$$

where $u(t)=u(t, x)=\left(u_{1}(t, x), \cdots, u_{n}(t, x)\right)$ with $n \geq 1, r_{\alpha}(t)=\nu t^{\alpha-1} / \Gamma(\alpha), \Gamma(\alpha)$ denotes the gamma function, $P(D)=\Delta_{x}$ is the Laplacian operator on $x$-variable, $\nu$ denotes the Newtonian viscosity and $f: \mathbb{R} \rightarrow \mathbb{R}$ is a function satisfying

$$
f(0)=0 \text { and }|f(a)-f(b)| \leq C|a-b|\left(|a|^{\rho-1}+|b|^{\rho-1}\right),
$$

here $\rho>1$ and $C$ is a positive constant independent of $a, b \in \mathbb{R}$. Typical examples of $f(u)$ are given by $\gamma|u|^{\rho-1} u$ and $\gamma|u|^{\rho}$ for $\gamma \in\{+,-\}$. This nonlinearities yield a scaling for (1) which is fundamental in our approach on Besov-Morrey spaces, modeled on Besov space, but with underlying norm is of Morrey type. This spaces have been introduced by H. Kozono and M. Yamazaki [4] for analysis of the Navier-Stokes equations. As far

\footnotetext{
${ }^{1}$ nucaltiado@gmail.com.br

2precioso@ibilce.unesp.br
} 
as we know, the problem of existence of solutions to (1) in Besov-Morrey space is new as $1<\alpha<2$. Formally the problem (1) is equivalent to (FPDE),

$$
\begin{aligned}
& \partial_{t}^{\alpha} u=\nu P(D) u+f(u) \quad \text { in }(0, \infty) \times \mathbb{R}^{n} \\
& u_{t}(0)=0 \text { and } u(0)=u_{0} \text { in } \mathbb{R}^{n},
\end{aligned}
$$

where $\partial_{t}^{\alpha} u=D_{0 \mid t}^{\alpha-1} u_{t}, u_{t}=\frac{\partial u}{\partial t}$ and $D_{0 \mid t}^{\alpha-1}$ stands for the Riemann-Liouville derivative of order $\alpha-1, D_{0 \mid t}^{\alpha-1} u=\frac{1}{\Gamma(2-\alpha)} \frac{\partial}{\partial t} \int_{0}^{t} \frac{u(s)}{(t-s)^{\alpha-1}} d s, \quad(t>0$ and $1<\alpha<2)$ for a suitable $u(\cdot, x) \in L_{l o c}^{1}\left(\mathbb{R}^{n}\right)$. Employing a Duhamel-type formula in (3)-(4) (or (1)), formally we obtain the integral equation

$$
u(t)=L_{\alpha}(t) u_{0}+B_{\alpha}(u)(t),
$$

where

$$
B_{\alpha}(u)(t)=\int_{0}^{t} L_{\alpha}(t-s)\left(\int_{0}^{s} r_{\alpha-1}(s-\tau) f(u(\tau)) d \tau\right) d s
$$

and $\left\{L_{\alpha}(t)\right\}_{t \geq 0}$ stands for convolution operators (or diffusion-wave operator) given by

$$
\widehat{L_{\alpha}(t) \varphi}(\xi)=E_{\alpha}\left(-t^{\alpha}|\xi|^{2}\right) \widehat{\varphi}(\xi)
$$

for every Schwartz function $\varphi \in \mathcal{S}\left(\mathbb{R}^{n}\right)$. Throughout this paper a mild solution for (3)(4) (or (1)) is a function $u(t, x)$ satisfying (5) fol all $x \in \mathbb{R}^{n}, t>0$ and $u(t, x) \rightarrow u_{0}$ in $\mathcal{S}^{\prime}\left(\mathbb{R}^{n}\right)$ as $t \rightarrow 0^{+}$, actually we showed the weak convergence on homogeneous Besov space $\dot{B}_{\infty, \infty}^{2 /(\rho-1)}$. Here $E_{\alpha}\left(-t^{\alpha}|\xi|^{2}\right)$ stands for Mittag-Leffler function and $\hat{\cdot}=\mathcal{F}$ stands for Fourier transform in Schwartz's space $\mathcal{S}\left(\mathbb{R}^{n}\right)$ which can be extended to tempered distributions' spaces $\mathcal{S}^{\prime}\left(\mathbb{R}^{n}\right)$. The operator $L_{\alpha}(t)$ does not satisfy the semigroup property $L_{\alpha}(t+s) \neq L_{\alpha}(t) L_{\alpha}(s)$ unless if $\alpha=1$. In this case, the operator $L_{1}(t)=S(t)$ is the heat semigroup, because $E_{1}\left(-t|\xi|^{2}\right)=e^{-t|\xi|^{2}}$. The kernel $k_{\alpha}$ of $L_{\alpha}(t)$ is the fundamental solution of $((3)$ with $f \equiv 0)$ given by

$$
k_{\alpha}(t, x)=\int_{\mathbb{R}^{n}} e^{i x \cdot \xi} E_{\alpha}\left(-t^{\alpha}|\xi|^{2}\right) d \xi .
$$

The FPDE (3)-(4) interpolate two groups of PDEs (see e.g. [2]), namely hyperbolic $(\alpha=2)$ and parabolic $(\alpha=1)$ PDEs, which have been widely investigated in the last years. Theses groups presents many differences in theory of the existence and asymptotic behavior of solutions in scaling invariant spaces. In the case $\alpha=1$, the FPDE (3) reduces to the usual semilinear heat equation which is well documented in singular spaces, see e.g. [4]. Without making a complete list, we mention the weak- $L^{p}$ spaces, the Besov spaces $\dot{B}_{p, \infty}^{s}$, the Morrey spaces $\mathcal{M}_{p, \mu}$, the $L^{p}$-spaces, the Besov-Morrey spaces $\mathcal{N}_{p, \mu, \infty}^{s}$ and so on. However, there are few papers dealing with FPDEs on those spaces. In [3], the authors based in Mihlin-Hörmander's theorem establish $L^{p}-L^{r}$ estimates to Mittag-Leffler's family (7) and local well-posedness is obtained in a $L^{r}\left(\mathbb{R}^{n}\right)$-framework. In [5], the authors showed the existence of self-similar global solution with initial data $u_{0} \in \dot{B}_{p, \infty}^{n / p-2 /(\rho-1)} \cap \mathcal{E}_{q\left(r, p_{0}\right), r}$. 
Our initial data $u_{0}$ (see Theorem 3.1) is larger than the previous works and can be taken as strongly singular function. Indeed, if $\mu=n-\frac{2}{\rho-1}$ and $\lambda=n-\frac{2 p}{\rho-1}$, we obtain the continuous inclusions

$$
L^{q} \subset \text { weak- } L^{q} \subset \mathcal{M}_{p, \lambda} \subset \mathcal{N}_{p, \mu, \infty}^{\sigma} \text { and } \quad \dot{B}_{r, \infty}^{k} \subset \mathcal{N}_{p, \mu, \infty}^{\sigma}
$$

provided $\frac{n}{q}=\frac{n-\lambda}{p}=-\sigma+\frac{n-\mu}{p}=-k+\frac{n}{r}$, where $\sigma=\frac{n-\mu}{p}-\frac{2}{\rho-1}, k=\frac{n}{r}-\frac{2}{\rho-1}$ and $1 \leq q \leq r \leq p<\frac{n(\rho-1)}{2}$ (all spaces in (8) are invariant by scaling (24)).

The aim of this work is to establish the existence of solutions for (3)-(4) in the framework of Besov-Morrey spaces.

\section{Preliminaries}

Let $D_{r}\left(x_{0}\right)$ be the open ball in $\mathbb{R}^{n}$ centered at $x_{0}$ and with radius $r>0$. Give two parameters $1 \leq p<\infty$ and $0 \leq \mu<n$, the Morrey spaces $\mathcal{M}_{p, \mu}=\mathcal{M}_{p, \mu}\left(\mathbb{R}^{n}\right)$ is defined to be the set of functions $f \in L^{p}\left(D_{r}\left(x_{0}\right)\right)$ such that

$$
\|f\|_{p, \mu}:=\sup _{x_{0} \in \mathbb{R}^{n}} \sup _{r>0} r^{-\frac{\mu}{p}}\|f\|_{L^{p}\left(D_{r}\left(x_{0}\right)\right)}<\infty
$$

which is a Banach space endowed with norm (9). For $s \in \mathbb{R}$ and $1 \leq p<\infty$, the homogeneous Sobolev-Morrey space $\mathcal{M}_{p, \mu}^{s}=(-\Delta)^{-s / 2} \mathcal{M}_{p, \mu}$ is the Banach space with norm

$$
\|f\|_{\mathcal{M}_{p, \mu}^{s}}=\left\|(-\Delta)^{s / 2} f\right\|_{p, \mu} .
$$

Taking $p=1$, we have $\|f\|_{L^{1}\left(D_{r}\left(x_{0}\right)\right)}$ denotes the total variation of $f$ on open ball $D_{r}\left(x_{0}\right)$ and $\mathcal{M}_{1, \mu}$ stands for space of signed measure s. In particular, $\mathcal{M}_{1,0}=\mathcal{M}$ is the space of finite measures. For $p>1$, we have $\mathcal{M}_{p, 0}=L^{p}$ and $\mathcal{M}_{p, 0}^{s}=\dot{H}_{p}^{s}$ is the well known Sobolev space. The space $L^{\infty}$ corresponds to $\mathcal{M}_{\infty, \mu}$. Morrey and Sobolev-Morrey spaces presents the following scaling

$$
\|f(\lambda \cdot)\|_{p, \mu}=\lambda^{-\frac{n-\mu}{p}}\|f\|_{p, \mu}
$$

and

$$
\|f(\lambda \cdot)\|_{\mathcal{M}_{p, \mu}^{s}}=\lambda^{s-\frac{n-\mu}{p}}\|f\|_{\mathcal{M}_{p, \mu}^{s}},
$$

where the exponent $s-\frac{n-\mu}{p}$ is called scaling index and $s$ is called regularity index. We have that

$$
(-\Delta)^{l / 2} \mathcal{M}_{p, \mu}^{s}=\mathcal{M}_{p, \mu}^{s-l} .
$$

Morrey spaces contain Lebesgue and weak- $L^{p}$, with the same scaling indexes. Precisely, we have the continuous proper inclusions

$$
L^{p}\left(\mathbb{R}^{n}\right) \varsubsetneqq \text { weak- } L^{p}\left(\mathbb{R}^{n}\right) \varsubsetneqq \mathcal{M}_{r, \mu}\left(\mathbb{R}^{n}\right)
$$


where $r<p$ and $\mu=n(1-r / p)$. Let $\mathcal{S}\left(\mathbb{R}^{n}\right)$ and $\mathcal{S}^{\prime}\left(\mathbb{R}^{n}\right)$ be the Schwartz space and the tempered distributions, respectively. Let $\varphi \in \mathcal{S}\left(\mathbb{R}^{n}\right)$ be nonnegative radial function such that

$$
\operatorname{supp}(\varphi) \subset\left\{\xi \in \mathbb{R}^{n} ; \frac{1}{2}<|\xi|<2\right\} \text { and } \sum_{j=-\infty}^{\infty} \varphi_{j}(\xi)=1, \text { for all } \xi \neq 0
$$

where $\varphi_{j}(\xi)=\varphi\left(2^{-j} \xi\right)$. Let $\phi(x)=\mathcal{F}^{-1}(\varphi)(x)$ and $\phi_{j}(x)=\mathcal{F}^{-1}\left(\varphi_{j}\right)(x)=2^{j n} \phi\left(2^{j} x\right)$ where $\mathcal{F}^{-1}$ stands for inverse Fourier transform. For $1 \leq q<\infty, 0 \leq \mu<n$ and $s \in \mathbb{R}$, the homogeneous Besov-Morrey space $\mathcal{N}_{q, \mu, r}^{s}\left(\mathbb{R}^{n}\right)\left(\mathcal{N}_{q, \mu, r}^{s}\right.$ for short) is defined to be the set of $u \in \mathcal{S}^{\prime}\left(\mathbb{R}^{n}\right)$, modulo the space of polynomials $\mathcal{P}$, such that $\mathcal{F}^{-1} \varphi_{j}(\xi) \mathcal{F} u \in \mathcal{M}_{q, \mu}$ for all $j \in \mathbb{Z}$ and

$$
\|u\|_{\mathcal{N}_{q, \mu, r}^{s}}= \begin{cases}\left(\sum_{j \in \mathbb{Z}}\left(2^{j s}\left\|\phi_{j} * u\right\|_{q, \mu}\right)^{r}\right)^{\frac{1}{r}}<\infty, & 1 \leq r<\infty \\ \sup _{j \in \mathbb{Z}} 2^{j s}\left\|\phi_{j} * u\right\|_{q, \mu}<\infty, & r=\infty .\end{cases}
$$

In particular $\mathcal{N}_{q, 0, r}^{s}=\dot{B}_{q, r}^{s}$ denotes the homogeneous Besov spaces and for $1 \leq q \leq p<\infty$ such that $\mu=n(1-q / p)$ we obtain $\mathcal{N}_{q, \mu, r}^{s} \equiv \mathcal{N}_{p, q, r}^{s}$, because $\mathcal{M}_{q, \mu}=\mathcal{M}_{q}^{p}$. The space $\mathcal{N}_{q, \mu, r}^{s}$ is a Banach space and have the real interpolation properties

$$
\mathcal{N}_{q, \mu, r}^{s}=\left(\mathcal{M}_{q, \mu}^{s_{1}}, \mathcal{M}_{q, \mu}^{s_{2}}\right)_{\theta, r}
$$

and

$$
\mathcal{N}_{q, \mu, r}^{s}=\left(\mathcal{N}_{q, \mu, r_{1}}^{s_{1}}, \mathcal{N}_{q, \mu, r_{2}}^{s_{2}}\right)_{\theta, r},
$$

for all $s_{1} \neq s_{2}, 0<\theta<1$ and $s=(1-\theta) s_{1}+\theta s_{2}$. Here $(X, Y)_{\theta, r}$ stands for the real interpolation space between $X$ and $Y$ constructed via the $K_{\theta, q}$-method. Recall that $(\cdot, \cdot)_{\theta, r}$ is an exact interpolation functor of exponent $\theta$ on the category of normed spaces.

In the next lemmas, we collect basic facts about Morrey spaces and Besov-Morrey spaces.

Lemma 2.1. Suppose that $s_{1}, s_{2} \in \mathbb{R}, 1 \leq p_{1}, p_{2}, p_{3}<\infty$ and $0 \leq \mu_{i}<n, i=1,2,3$.

(i) (Inclusion) If $\frac{n-\mu_{1}}{p_{1}}=\frac{n-\mu_{2}}{p_{2}}$ and $p_{2} \leq p_{1}$,

$$
\mathcal{M}_{p_{1}, \mu_{1}} \subset \mathcal{M}_{p_{2}, \mu_{2}} \text { and } \mathcal{N}_{p_{1}, \mu_{1}, 1}^{0} \subset \mathcal{M}_{p_{1}, \mu_{1}} \subset \mathcal{N}_{p_{1}, \mu_{1}, \infty}^{0} .
$$

(ii) (Sobolev-type embedding) Let $j=1,2$ and $p_{j}, s_{j}$ be $p_{2} \leq p_{1}, s_{1} \leq s_{2}$ such that $s_{2}-$ $\frac{n-\mu_{2}}{p_{2}}=s_{1}-\frac{n-\mu_{1}}{p_{1}}$, we obtain

$$
\mathcal{M}_{p_{2}, \mu}^{s_{2}} \subset \mathcal{M}_{p_{1}, \mu}^{s_{1}},\left(\mu=\mu_{1}=\mu_{2}\right)
$$

and for every $1 \leq r_{2} \leq r_{1} \leq \infty$, we have

$$
\mathcal{N}_{p_{2}, \mu_{2}, r_{2}}^{s_{2}} \subset \mathcal{N}_{p_{1}, \mu_{1}, r_{1}}^{s_{1}} \quad \text { and } \quad \mathcal{N}_{p_{2}, \mu_{2}, r_{2}}^{s_{2}} \subset \dot{B}_{\infty, r_{2}}^{s_{2}-\frac{n-\mu_{2}}{p_{2}}} .
$$


(iii) (Hölder inequality) Let $\frac{1}{p_{3}}=\frac{1}{p_{2}}+\frac{1}{p_{1}}$ and $\frac{\mu_{3}}{p_{3}}=\frac{\mu_{2}}{p_{2}}+\frac{\mu_{1}}{p_{1}}$. If $f_{j} \in \mathcal{M}_{p_{j}, \mu_{j}}$ with $j=1,2$, then $f_{1} f_{2} \in \mathcal{M}_{p_{3}, \mu_{3}}$ and

$$
\left\|f_{1} f_{2}\right\|_{p_{3}, \mu_{3}} \leq\left\|f_{1}\right\|_{p_{1}, \mu_{1}}\left\|f_{2}\right\|_{p_{2}, \mu_{2}} .
$$

We performed a scaling analysis in order to choose the correct indexes of spaces such that their norms are invariant by scaling (23). Indeed, it is well known that if $u$ solves (3) with $f(u)=\gamma|u|^{\rho-1} u$ then, for each $\lambda>0$, the rescaled function $u_{\lambda}(t, x)=\lambda^{\frac{2}{\rho-1}} u\left(\lambda^{\frac{2}{\alpha}} t, \lambda x\right)$ is also a solution. This led us to define a scaling map for (3) as

$$
u(t, x) \mapsto u_{\lambda}(t, x) .
$$

Making $t \rightarrow 0^{+}$in (23) this map induces the following scaling for initial data $u_{0}(x)$,

$$
u_{0}(x) \mapsto u_{0 \lambda}(x)=\lambda^{\frac{2}{\rho-1}} u_{0}(\lambda x) .
$$

Let $B C((0, \infty), X)$ be the class of the bounded functions from $(0, \infty)$ into a Banach space $X$. We define our ambient spaces based on Besov-Morrey type spaces as follows

$$
X_{q}^{p}=\left\{u \in B C\left((0, \infty) ; \mathcal{N}_{p, \mu, \infty}^{\sigma}\right): t^{\eta} u \in B C\left((0, \infty) ; \mathcal{M}_{q, \mu}\right)\right\},
$$

which endowed with norm

$$
\|u\|_{X_{q}^{p}}:=\sup _{t>0}\|u(t, \cdot)\|_{\mathcal{N}_{p, \mu, \infty}^{\sigma}}+\sup _{t>0} t^{\eta}\|u(t, \cdot)\|_{\mathcal{M}_{q, \mu}}
$$

is a Banach space for $(1<p \leq q<\infty)$. Here $\eta \in \mathbb{R}$ and $\sigma<0, \eta=\frac{\alpha}{2}\left(\frac{2}{\rho-1}-\frac{n-\mu}{q}\right)$ and $\sigma=$ $\frac{n-\mu}{p}-\frac{2}{\rho-1}$ having been chosen such that the norm (26) is invariant by scaling map (23).

\section{Results}

Theorem 3.1 (Well-posedness). Let $n \geq 1$ and $1 \leq \alpha<2$ and let $1<\{\rho, p\} \leq q<\infty$, $0 \leq \mu<n$ be such that $\frac{2}{\rho-1}-\frac{2}{\alpha \rho}<\frac{n-\mu}{q}<\frac{2}{\alpha(\rho-1)}$ and $\frac{n-\mu}{p}<\frac{2}{\rho-1}$.

(i) (Existence and uniqueness) Let $\varepsilon>0$ and $\delta=\delta(\varepsilon)$ be such that $\left\|u_{0}\right\|_{\mathcal{N}_{p, \mu, \infty}^{\sigma}} \leq \delta$. Then the problem (1) has a mild solution $u \in X_{q}^{p}$ which is unique, if u lies in closed small balls $D_{r} \subset X_{q}^{p}, r<2 \varepsilon$. Also, $u(t) \rightarrow u_{0}$ in the weak-* topology of $\dot{B}_{\infty, \infty}^{2 /(\rho-1)}$ as $t \rightarrow 0^{+}$.

(ii) (Continuous dependence on data) Let $D_{\delta} \subset \mathcal{N}_{p, \mu, \infty}^{\sigma}$ be closed ball and let $u \in X_{q}^{p}$ be a mild solution associated to data $u_{0} \in D_{\delta}$. Then data-solution map $u_{0} \in D_{\delta} \longmapsto$ $u \in X_{q}^{p}$ is Lipschitz continuous.

We start by recalling an elementary fixed point lemma whose proof can be found in $[1]$. 
Lemma 3.1. Let $(X,\|\cdot\|)$ be a Banach space and $1<\rho<\infty$. Suppose that $B: X \rightarrow X$ satisfies $B(0)=0$ and

$$
\|B(x)-B(z)\| \leq K\|x-z\|\left(\|x\|^{\rho-1}+\|z\|^{\rho-1}\right) .
$$

Let $R>0$ be the unique positive root of $2^{\rho} K R^{\rho-1}-1=0$. Given $0<\varepsilon<R$ and $y \in X$ such that $\|y\| \leq \varepsilon$, there exists a solution $x \in X$ for the equation $x=y+B(x)$ which is the unique one in the closed ball $D_{2 \varepsilon}=\{z \in X ;\|z\| \leq 2 \varepsilon\}$. Moreover, if $\|\bar{y}\| \leq \varepsilon$ and $\bar{x} \in D_{2 \varepsilon}$ satisfies the equation $\bar{x}=\bar{y}+B(\bar{x})$ then

$$
\|x-\bar{x}\| \leq \frac{1}{1-2^{\rho} K \varepsilon^{\rho-1}}\|y-\bar{y}\| .
$$

The integral equation (5) has the form $u=y+B(u, u)$ on the space $X=X_{q}^{p}$ where $y=L_{\alpha}(t) u_{0}$ and $B(u, u)$ is given by (32). We invoke the Lemma 3.1 in our proof, hence the estimates for linear and nonlinear part of (5) will be necessary. However, to obtain them first we need to derive estimates for the Mittage-Leffer convolution operators $\left\{L_{\alpha}(t)\right\}_{t \geq 0}$ on Sobolev-Morrey spaces and Besov-Morrey spaces.

Lemma 3.2. Let $s, \beta \in \mathbb{R}, 1 \leq \alpha<2,1<p \leq q<\infty, 0 \leq \mu<n$, and $(\beta-s)+\frac{n-\mu}{p}-$ $\frac{n-\mu}{q}<2$ where $\beta \geq s$.

(i) There exists $C>0$ such that

$$
\left\|L_{\alpha}(t) f\right\|_{\mathcal{M}_{q, \mu}^{\beta}} \leq C t^{-\frac{\alpha}{2}(\beta-s)-\frac{\alpha}{2}\left(\frac{n-\mu}{p}-\frac{n-\mu}{q}\right)}\|f\|_{\mathcal{M}_{p, \mu}^{s}},
$$

for every $t>0$ and $f \in \mathcal{M}_{p, \mu}^{s}$.

(ii) Let $r \in[1, \infty]$, there exists $C>0$ such that

$$
\left\|L_{\alpha}(t) f\right\|_{\mathcal{N}_{q, \mu, r}^{\beta}} \leq C t^{-\frac{\alpha}{2}(\beta-s)-\frac{\alpha}{2}\left(\frac{n-\mu}{p}-\frac{n-\mu}{q}\right)}\|f\|_{\mathcal{N}_{p, \mu, r}^{s}},
$$

for every $f \in \mathcal{S}^{\prime} / \mathcal{P}$ and $t>0$.

(iii) Let $r \in[1, \infty]$ and $\beta>s$, there exists $C>0$ such that

$$
\left\|L_{\alpha}(t) f\right\|_{\mathcal{N}_{q, \mu, 1}^{\beta}} \leq C t^{-\frac{\alpha}{2}(\beta-s)-\frac{\alpha}{2}\left(\frac{n-\mu}{p}-\frac{n-\mu}{q}\right)}\|f\|_{\mathcal{N}_{p, \mu, r}^{s}},
$$

for every $f \in \mathcal{S}^{\prime} / \mathcal{P}$.

Lemma 3.3. Under the assumptions of the Theorem 3.1, there exists $L>0$ such that

$$
\left\|L_{\alpha}(t) u_{0}\right\|_{X_{q}^{p}} \leq L\left\|u_{0}\right\|_{\mathcal{N}_{p, \mu, \infty}^{\sigma}}
$$

for all $u_{0} \in \mathcal{N}_{p, \mu, \infty}^{\sigma}$. Let $s=2 /(\rho-1)$, if $u_{0} \in \dot{B}_{\infty, \infty}^{s}$ we obtain $L_{\alpha}(t) u_{0} \rightarrow u_{0}$ in the weak-* topology of $\dot{B}_{\infty, \infty}^{s}$ as $t \rightarrow 0^{+}$.

Lemma 3.4 (Nonlinear estimate). Under the assumptions of Theorem 3.1. There is a positive constant $K$ such that

$$
\left\|B_{\alpha}(u)-B_{\alpha}(v)\right\|_{X_{q}^{p}} \leq K\|u-v\|_{X_{q}^{p}}\left(\|u\|_{X_{q}^{p}}^{\rho-1}+\|v\|_{X_{q}^{p}}^{\rho-1}\right),
$$

for $K=K_{1}+K_{2}$. Moreover, we have $B_{\alpha}(u)(t) \rightarrow 0$ in the weak-* topology of $\dot{B}_{\infty, \infty}^{2 /(\rho-1)}$ as $t \rightarrow 0^{+}$. 


\subsection{Proof of Theorem 3.1}

Let $0<\varepsilon<R=\left(1 / 2^{\rho} K\right)^{\rho-1}$, where $K>0$ and $L>0$ are the constants obtained in Lemma 3.3 and Lemma 3.4, respectively. Let $\delta=\varepsilon / L$, the Lemma 3.1 with $X=X_{q}^{p}$ and $y=L_{\alpha}(t) u_{0}$ yields the existence of an unique global mild solution $u \in X_{q}$ such that $\|u\|_{X_{q}} \leq \varepsilon$. Moreover, the Lemmas 3.3 and 3.4 yield $u(t) \rightarrow u_{0}$ in the weak-* topology of $\dot{B}_{\infty, \infty}^{2 /(\rho-1)}$ as $t \rightarrow 0^{+}$. The dependence of the initial data can be obtained from Lemma 3.3 and Lemma 3.1. Indeed, let $\bar{y}=L_{\alpha}(t) \overline{u_{0}}$ where $\overline{u_{0}} \in \mathcal{N}_{p, \mu, \infty}^{\sigma}$, then

$$
\begin{aligned}
\|u(t)-\bar{u}(t)\|_{X_{q}^{p}} & \leq \frac{1}{1-2^{\rho} K \varepsilon^{\rho-1}}\left\|L_{\alpha}(t)\left(u_{0}-\overline{u_{0}}\right)\right\|_{X_{q}} \\
& \leq \frac{1}{1-2^{\rho} K \varepsilon^{\rho-1}}\left\|u_{0}-\bar{u}_{0}\right\|_{\mathcal{N}_{p, \mu, \infty}^{\sigma}} .
\end{aligned}
$$

\section{References}

[1] L.C.F. Ferreira and E. J. Villamizar-Roa, Self-similar solutions, uniqueness and longtime asymptotic behavior for semilinear heat equations, Differential Integral Equations 19, no. 12, 1349-1370, 2006.

[2] G. Gripenberg, Weak solutions of hyperbolic-parabolic Volterra equations, Trans. Amer. Math. Soc. 343, no. 2, 675-694, 1994.

[3] H. Hirata and C. Miao, Space-time estimates of linear flow and application to some nonlinear integro-differential equations corresponding to fractional-order time derivative, Adv. Differential Equations 7, no. 2, 217-236, 2002.

[4] H. Kozono, M. Yamazaki, Semilinear heat equations and the Navier-Stokes equation with distributions in new function spaces as initial data, Comm. Partial Differential Equations 19, no. 5-6, 959-1014, 1994. DOI:10.1080/03605309408821042

[5] C.X. Miao, H. Yang, The self-similar solution to some nonlinear integro-differential equations corresponding to fractional order time derivative. Acta Math. Sin. (Engl. Ser.) 21, no. 6, 1337-1350, 2005. DOI: 10.1007/s10114-005-05-16-0 